\title{
Home-based unsupervised pulmonary rehabilitation program improves the respiratory disability in systemic sclerosis patients with dyspnea: an observational prospective study
}

\author{
Silvia Faverzani ${ }^{1}$, Filippo Nocera ${ }^{2}$, Ernesto Crisafulli ${ }^{3}$, Maurizio Marvisi ${ }^{4}$, Andrea Becciolini ${ }^{2}$, \\ Federica Mosetti ${ }^{1}$, Eleonora Di Donato ${ }^{2}$, Flavio Mozzani ${ }^{2}$, Daniele Santilli ${ }^{2}$, Giuseppe Scopelliti ${ }^{5}$, \\ Alarico Ariani ${ }^{2}$ \\ ${ }^{1}$ Rehabilitation Medicine Service, Rehabilitation Geriatrics Department, University Hospital of Parma; ${ }^{2}$ Department of \\ Medicine, Internal Medicine and Rheumatology Unit, University Hospital of Parma; ${ }^{3}$ Department of Medicine, Respiratory \\ Medicine Unit, University of Verona and Azienda Ospedaliera Universitaria Integrata of Verona; ${ }^{4}$ Internal Medicine Unit, \\ Figlie di San Camillo Hospital, Cremona; ${ }^{5}$ Cystic Fibrosis Unit, Azienda Ospedaliero Universitaria di Ancona, Italy
}

\begin{abstract}
Dyspnea is a common symptom in systemic sclerosis (SSc) that considerably decreases patients' quality of life (QoL). Pulmonary rehabilitation (PR) mitigates dyspnea impact on daily activities.
\end{abstract}

Correspondence: Silvia Faverzani, University of Parma, University Hospital of Parma, Viale A. Gramsci 14, 43126 Parma, Italy. Tel. +39.338.3631798.

E-mail: silvia.faverzani@unipr.it

Key words: Pulmonary rehabilitation; systemic sclerosis; dyspnea.

Contributions: SF, FN, assessed, trained and gave instructions to the patients, collected dat; AB, EDD, FM, DS, enrolled patients; MM, EC, $\mathrm{AB}, \mathrm{AA}, \mathrm{GS}$, made statistical analysis; GS, wrote the assessment and training protocols; $\mathrm{AA}, \mathrm{AB}, \mathrm{SF}, \mathrm{FN}$, designed the study; $\mathrm{AA}, \mathrm{AB}$, analyzed the data; SF, manuscript drafting. All the authors read and approved the final version of the manuscript and agreed to be accountable for all aspects of the work.

Conflict of interest: The authors declare that they have no competing interests, and all authors confirm accuracy.

Ethics approval and consent to participate: The study was approved by the Ethical Committee of "Area Vasta Emilia Nord" (Protocol n. 198/04012021). All patients provided written and informed consent.

Received for publication: 21 June 2021.

Accepted for publication: 17 December 2021.

Publisher's note: All claims expressed in this article are solely those of the authors and do not necessarily represent those of their affiliated organizations, or those of the publisher, the editors and the reviewers. Any product that may be evaluated in this article or claim that may be made by its manufacturer is not guaranteed or endorsed by the publisher.

${ }^{\circ}$ Copyright: the Author(s), 2021

Licensee PAGEPress, Italy

Monaldi Archives for Chest Disease 2022; 92:1984

doi: 10.4081/monaldi.2021.1984

This article is distributed under the terms of the Creative Commons Attribution-NonCommercial International License (CC BY-NC 4.0) which permits any noncommercial use, distribution, and reproduction in any medium, provided the original author(s) and source are credited.
The aim of this study is to evaluate the effect on respiratory disability of home-based PR in SSc patients with dyspnea. In this observational prospective monocentric study, we screened all dyspneic SSc consecutive patients attending the Rheumatological day hospital in the University hospital of Parma from January 2019 and June 2019. The aim of our study was to understand if a PR unsupervised home-based program could improve respiratory disability in this specific population. Dyspnea was evaluated with the self-administered questionnaires modified Medical Research Council (mMRC) and Saint George's Respiratory Questionnaire (SGRQ). Patients also filled in Short Form 36 (SF36) and the Modified-Health Assessment Questionnaire for SSc (HAQ-MOD). Health Professionals assessed and trained the patients and collected data before PR and at the end of the program. PR consisted in 5 weekly unsupervised sessions for 8 weeks. Wilcoxon test for paired data evaluated the changes after PR. $p<0.05$ was considered statistically significant. $46 \mathrm{SSc}$ patients were included (43 female). Only 31 (29 female) performed PR as planned (Adherent GroupAG) while the others gave up within the first week (Non-Adherent Group-NAG). All SGRQ domains (symptoms: from 30 to 18; $\mathrm{p}=0.0055$; activity: from 47 to $35, \mathrm{p}=0.23$; impact from 29 to 25 , $\mathrm{p}=0.044$ ) and SGRQ total score (from 35 to $29 ; \mathrm{p}=0.022$ ) improved in AG. SGRQ scores did not change in NAG as well as SF36 and HAQ-MOD in both groups. The home-based PR program dramatically decreased the effect, frequency and severity of respiratory symptoms. Conversely, it slightly changed the activities causing breathlessness and dyspnea-related social functioning disturbances. PR appears to be a useful tool in treatment strategies aiming to achieve a QoL improvement in SSc patients.

\section{Introduction}

Systemic sclerosis (SSc) is a rare rheumatic disease, characterized by frequent involvement of the lungs with interstitial lung disease (ILD) and PAH (pulmonary arterial hypertension). Dyspnea on exertion and at rest, fatigue and exercise intolerance are the main respiratory disabling symptoms.

SSc patients with lung impairment have low physical activity levels and report impaired quality of life (QoL). Dyspnea is related to a decrease of function and QoL as well as exercise tolerance is associated with reduced QoL and poor survival [1].

Pulmonary rehabilitation (PR) is a patient-tailored global 
intervention that includes exercise training, education, and behavior change. Its aim is to improve the physical and psychological condition of people with chronic respiratory disease [2]. The American Thoracic Society and European Respiratory Society consensus report supports PR in patients affected by interstitial lung disease (ILD). PR, provided to individuals with chronic respiratory diseases other than COPD, has demonstrated improvements in symptoms, exercise tolerance, and QoL [3].

Respiratory disability is common in systemic sclerosis (SSc), but so far, at the best of our knowledge, no studies have investigated the PR impact in this specific population. Therefore, the first aim of this study is to investigate if a PR program in SSc patients with dyspnea can improve respiratory disability.

\section{Methods}

This observational prospective study was carried out following the Declaration of Helsinki and approved by the local Ethic Committee. The principal aim of this research was to evaluate with questionnaires if respiratory symptoms and disability could improve with a non-supervised home-based PR program.

\section{Patient reported outcomes}

SGRQ is a standardized self-administered disease-specific instrument. It measures impact on overall health, daily life, and perceived well-being in patients with lung chronic diseases. It consists of 76 items divided into three parts measuring "symptoms" (distress caused by respiratory symptoms), "activity" (disturbance for limitation in daily activities) and "impact" (social and emotional impact of disease). Scores range from zero (no impairment) to 100 (maximum impairment): a higher score means a poorer quality of life [4]. A decrease of 4 units, after a medical or non-medical intervention, in the SGRQ score is generally accepted in the literature to be a valid minimal important difference (MID) of beneficial treatment [5].

The HAQ-DI is a self-reported questionnaire with 8 domains of activities (dressing, arising, eating, walking, hygiene, reach, grip, and common daily activities). For each item, patients report the amount of difficulty during the activity. The HAQ DI is recommended for use in trials to evaluate change over time in groups of SSc patients [6,7]. The SF36 is a short questionnaire and an indicator of overall health status. It consists of 36 items Scores range from 0 (worst possible health state and more disability) to 100 (best possible health state and less disability) [8]. The mMRC scale is a self-rating tool to measure the degree of disability that breathlessness poses on day-to-day activities on a scale from 0 to 4 . An increasing mMRC score reflects impaired QoL and a high respiratory symptoms burden.

\section{Patients}

All the SSc patients, diagnosed according to ACR/EULAR criteria and followed at University Hospital of Parma, fill in SGRQ, HAQ-DI, SF36, mMRC, during their medical examination. The health professionals collected data and offered all the dispneic subjects $(\mathrm{mMRC}>0)$ a $\mathrm{PR}$ program they could perform at home. Patients who refused to participate, with $\mathrm{mMRC}=0$ and/or with recent pulmonary exacerbation were excluded. The two groups were not randomized but we decided to compare the subjects who completed the whole program (Adherent Group-AG) and the NonAdherent Group (NAG). Changes in the outcomes were compared between the 2 groups.
Patients have been evaluated with field tests, Six Minutes Step Test-6MST and 30 Seconds Sit to Stand Chair Test-30"STSCT, at the beginning and at the end of the study, to assess exercise tolerance: the physiotherapist collected hearth and respiratory rate, blood pressure, oxygen saturation, the values of dyspnea and muscular fatigue with the Borg scale before and after every test, the number of step after the 6MST and the number of sit to stand after the 30"STSCT. Patients also performed a modified Arm Curl Test $(\mathrm{mACT})$ with a $1.5 \mathrm{~kg}$ weight and with a resistance band: the health professional detected the number of repetitions, to assess the upper limbs training. The resistance of the elastic was the same for all of the subjects and we tested both arms. The program included 8 weeks of treatment, with 5 weekly sessions: 3 Interval Training (IT) sessions with a stepper and 2 sessions to improve upper limbs strength with weights and resistance bands. The home sessions were performed at home with no supervision: the step IT program started with a duration of $20 \mathrm{~min}$ and 1:1 rate and at the 8 th week had to achieve a duration of $40 \mathrm{~min}$; the strength upper limb training started with 1 set of 10 repetitions for every exercise, to achieve 3 sets of 10 repetitions for every exercise.

Patients learnt to achieve the overload weekly, by increasing of 5 repetitions per exercise every week, giving attention to subjective dyspnea and muscle fatigue. They were taught to use the Borg scale and not overcome the value of 6 . point. At the end of this initial assessment stage, the physiotherapist gave the patients two daily diaries (for IT sessions and for the upper limbs) and guidance material with figures and explanation of the upper limbs exercises. The health professionals collected questionnaires before and after PR to rate patient reported outcomes: mMRC, SGRQ, HAQ-DI, Scleroderma HAQ (SHAQ) and SF 36.

Patients were not supervised during the whole program, but they were re-evaluated with the same questionnaires and tests at the end of the 8 weeks. The physiotherapist also asked the patients if they had performed the whole program and checked the daily diaries to control the adherence of the patients.

\section{Statistical analysis}

Descriptive data were presented by medians (interquartile range) for continuous data or as numbers (percentages) for categorical data. Mann-Whitney test or Chi-square test investigated subgroups' differences as appropriate. Wilcoxon test for paired data evaluated the differences before and after the 8 weeks of PR. Statistical analyses were performed using Medcalc statistical software, version 18.2.1; $\mathrm{p}<0.05$ was considered statistically significant.

\section{Results}

Forty-six SSc patients with dyspnea were identified. 31 of enrolled patients performed the training as planned (adherent groupAG). Fifteen patients discontinued PR within the first week (non-adherent group-NAG). Patients basal (i.e., before PR) characteristics are in Table 1 . The two subgroups did not differ in terms of sex, ages, autoimmune profile, disease duration, pulmonary function, prevalence of interstitial lung disease and most of patients' reported outcomes (SGRQ scores, HAQ-MOD and SF36 physical component). Only the SF36 mental component was statistically different ( $30 v s 48 ; \mathrm{p}=0.002$ ). At the end of the program, in $\mathrm{AG}$ group the SGRQ total score decreased (from 35 to 29; $\mathrm{p}=0.022$ ) (Figures 1 and 2) as well as all SGRQ domains from t0 to t1. A statistically significant improvement was both in Symptoms and Impact scores (respectively from 30 to $18 ; \mathrm{p}=0.0055$ and from 29 
to $25, \mathrm{p}=0.044$ ). Similarly, the Activity score changed (from 47 to $35)$, without achieving the statistical significance $(\mathrm{p}=0.23)$. There were not any significant variations from $t 0$ and $t 1$ of HAQ-MOD (from 0.7 IQR 0.3-1.5 to 0.5 IQR 0.1-1.1; $\mathrm{p}=0.07$ ), SF36 physical component (from 33 IQR 28-42 to 38 IQR 30-45; $\mathrm{p}=0.38$ ) and SF36 mental component (from 48 IQR 41-51 to 48 IQR 39-55; $\mathrm{p}=0.15$ ).

In NAG, there were not modifications from to and $t 1$ of all

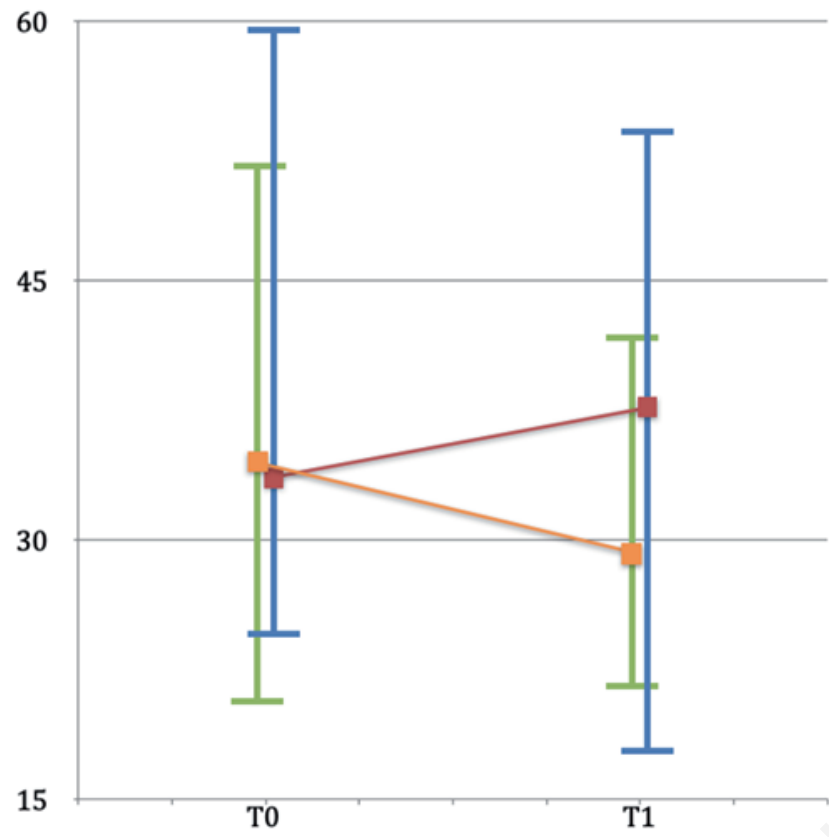

Figure 1. SGRQ total score change in adherent group (green/light line) and non-adherent group (blue/dark line) from to and $t 1$.
SGRQ scores (total: from 34 IQR 25-59 to 38 IQR 18-54; $\mathrm{p}=0.30$; symptoms: from 36 IQR 21-67 to 26 IQR 11-68; $p=0.25$; impact: from 28 IQR 19-48 to 33 IQR 10-46; $\mathrm{p}=0.36$; Activity: from 49 IQR 30-73 to 44 IQR 31-65; $\mathrm{p}=0.43$ ), HAQ-MOD (from 1.1 IQR 0.32.5 to 1.1 IQR $0.4-2.0 ; \mathrm{p}=0.42$ ) and SF36 (physical component: from 25 IQR 21-38 to 30 IQR 21-42; $\mathrm{p}=0.38$; mental component: from 30 IQR 22-39 to 31 IQR 23-41; $=0.56$ ) (Table 2).

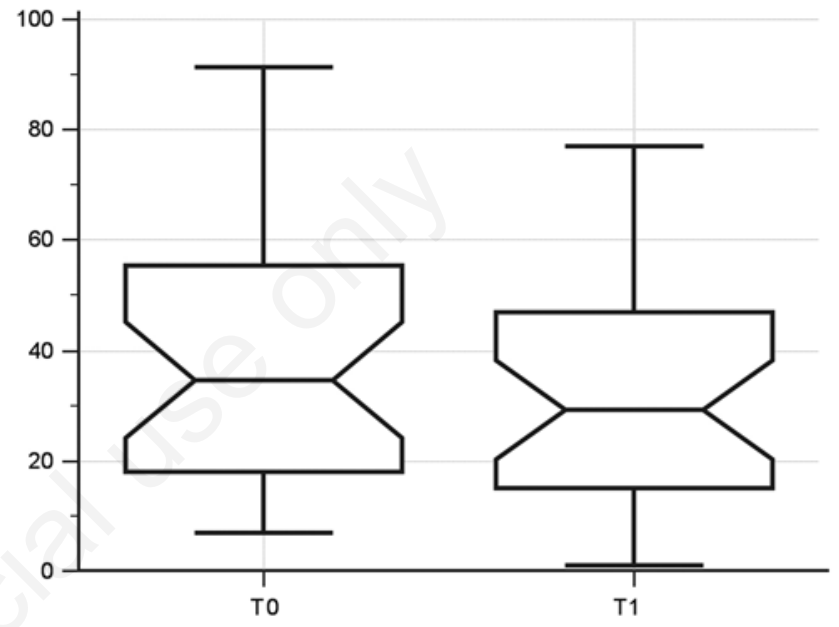

Figure 2. The notched box-and-whiskers plot of SGRQ score before (t0) and after (t1) PR treatment.

Table 1. Cohort characteristics.

\begin{tabular}{|c|c|c|c|c|}
\hline & Total cohort & Adherent group & Non-adherent group & p-value \\
\hline $\mathrm{N}$ & 46 & 31 & 15 & - \\
\hline Sex (M:F) & $3: 43$ & $2: 29$ & 1:14 & ns \\
\hline Age (median), yrs & $68(54-72)$ & $68(53-72)$ & $68(54-61)$ & ns \\
\hline Disease duration, yrs & $12(5-23)$ & $11(7-25)$ & $14(3-21)$ & ns \\
\hline AntiScl70, n (\%) & $14(30)$ & $12(39)$ & $2(13)$ & ns \\
\hline Anticentromere, n (\%) & $16(35)$ & $9(29)$ & $7(47)$ & ns \\
\hline FVC, \% (IQR) & $110(89-124)$ & $113(98-128)$ & $104(85-116)$ & ns \\
\hline $\mathrm{FEV}_{1}, \%(\mathrm{IQR})$ & $101(81-115)$ & $106(91-122)$ & $90(79-105)$ & ns \\
\hline DLco, \% (IQR) & $74(55-84)$ & $71(55-82)$ & $75(61-84)$ & ns \\
\hline TLC, \% (IQR) & $101(81-116)$ & $106(80-117)$ & $97(83-108)$ & ns \\
\hline ILD presence, n (\%) & $26(57)$ & $19(61)$ & $7(47)$ & ns \\
\hline T0 SGRQ score (IQR) & $34.2(19.7-56.2)$ & $34.8(18.0-55.4)$ & $33.6(25.1-59.7)$ & ns \\
\hline T0 SGRQ symptoms score (IQR) & $32.5(20.4-61.7)$ & $29.5(19.0-54.5)$ & $36.1(20.8-66.5)$ & ns \\
\hline T0 SGRQ activity score (IQR) & $47.2(29.3-72.3)$ & $47.2(29.3-66.1)$ & $49.5(29.9-72.7)$ & ns \\
\hline T0 SGRQ impact score (IQR) & $28.0(14.9-54.0)$ & $29.4(10.7-58.5)$ & $27.7(19.2-66.5)$ & ns \\
\hline T0 HAQ-MOD (IQR) & $0.875(0.250-1.600)$ & $0.700(0.250-1.475)$ & $1.125(0.281-2.531)$ & ns \\
\hline T0 SF36 - physical component & $32.0(25.0-40.0)$ & $33.0(28.3-41.8)$ & $25.0(21.0-38.0)$ & ns \\
\hline T0 SF36 - mental component & $42.5(32.0-51.0)$ & $48.0(41.0-51.0)$ & $30.0(21.5-39.0)$ & 0.002 \\
\hline
\end{tabular}

FVC, forced vital capacity (expressed in \% of the theoretical); $\mathrm{FEV}_{1}$, forced expiratory volume $1^{\text {st }}$ second (in \% of the theoretical); DLco, diffusion lung carbon monoxide (in \% of the theoretical); TLC, total lung capacity (in \% of the theoretical); ILD, interstitial lung disease; SGRQ, Saint George's Respiratory Questionnaire; HAQ-MOD, modified Health Assessment Questionnaire for SSc; SF36, Short Form 36; AG, adherent group; NAG, non-adherent Group; ns, not significant. 


\section{Discussion}

Exercise capacity in patients with ILD secondary to SSc is often limited by exertional and at rest dyspnea [9]. PR reduce breathlessness, with exercise training and education in lifestyle changes [2]. Exercise training is a mainstay of PR and it's considered the most useful way to optimize muscle performance in patients with respiratory disability [2]. All patients will benefit, independently of the severity of their illness [2].

No studies focused on PR in SSc patients. There are searches on PR in ILD patients, but most of the patients enrolled are affected by pulmonary fibrosis related to other systemic diseases [3]. Most of PR centers use an outpatient supervised model but there are lots of access barriers. So, home-based rehabilitation could be an alternative model to improve uptake and access. Appropriately resourced home-based exercise training has proven to be effective in reducing dyspnea and increasing exercise performance in individuals with chronic respiratory diseases $[10,11]$.

The PR program offered to SSc dyspneic patients improved respiratory disability: SGRQ total score decreased of 6 points with an important change in patient's well-being. Patients who performed the whole program had improvements in breathlessness and there was a statistical significance in symptoms and impact domains, so the patients had less perceived distress caused by dyspnea and a decrease in the effects of their respiratory disease on social and emotional daily life, according to the SGRQ score. A statistically difference between SF36 mental condition score in patients who dropped out the program has been found: these subjects had a worse psychological condition at baseline. Anxiety and depression are known to occur frequently in patients with dyspnea as ILDs: these symptoms are present in up to $50 \%$ [12] and $30 \%$ [13] of IPF patients. Depression in this context means symptoms of resignation and the physical performance of these patients is often reduced because of fear of breathlessness perception. Home-based PR may reduce anxiety, depression and psychological health by breaking the vicious circle of negative emotions, unpleasant breathing sensations and poor exercise performance [14].

There are some limits in this research. The adherence to the PR program was evaluated only by asking the patients if they had performed it and controlling the daily diaries they had to fill in. The reasons of non-adherence were not investigated unfortunately. The subjects were not randomized because they all needed PR training and it could be against ethical behavior not to propose it. The NAG seems to have worse respiratory function tests but there were no statistical or clinical differences as shown in the table. Patients were not screened with scales focused on anxiety and depression (e.g., the Hospital Anxiety and Depression Scale-HADS), to better understand the psychological impact of SSc in daily life. The lack of a constant supervision by the physiotherapist could be the first cause of drop out from the program. Maybe the NAG needed to be encouraged repeatedly by the health professional, to take up physical activity, more than the AG. Probably they had to be controlled and motivated frequently, also because of their higher mood problems at baseline. Patient's own motivation is important, but health professionals have to help patients to increase it. It could be also useful to identify activities that patients like doing, suitable for them, to improve adherence with the training in everyday life. So, systematic controls and supervision could be important for the next studies.

The follow up at six months and after one year were not evaluated: next studies need follow up data, because improvements achieved during PR do not automatically trigger a change in everyday life behaviors. Patients need to be re-evaluated to understand if gains are maintained after PR. A home-based rehabilitation PR model was used although the traditional centerbased one is highly effective in clinical trials [15]. In clinical practice, there is poor adherence because of some general barriers to uptake: health system inefficiencies, absence of a PR center, low referral rates, low knowledge about PR. There are also frequent patient-related barriers: transport to the center; distance from it, comorbidities and exacerbations [16]. Home-based programs have the potential to overcome many of these limitations.

There are some strengths in this research: the enrollment of patients who are not typical of PR delivery and the proposal of a low-cost program with no expensive equipment and home visits of health professionals.

QoL reduction and disability in SSc patients are only related to respiratory subjective impairment [17]. Short-term improvements of respiratory disability in SSc patients were observed: breathlessness and its impact on daily life, the main outcomes of $\mathrm{PR}$, were reduced. PR programs are an important opportunity to obtain behavior change and collaborative self-management also in SSc. The results of this research show that this PR model, with minimal resources, in terms of specialized equipment (water bottles/bands for resistance exercises and stairs for aerobic training) can be successfully delivered. Exercises and self-monitoring were performed at home, with no visits from a physiotherapist: the cost of our PR program for the health system was almost nil. It will be useful to replicate this home-based model with the same equipment but supervised by the physiotherapist. For example, weekly goals and telephone weekly appointments can encourage patients to fix new targets.

This study shows that PR should be part of a comprehensive intervention on SSc patients. Improvements from a psychological point of view were highlighted and these results focus on the importance to have also a specialized psychologist in the rehabilitation team. It should be better to increase the number of patients

Table 2. The results in AG and NAG groups before (t0) and after (t1) PR treatment.

\begin{tabular}{|c|c|c|c|c|c|c|}
\hline & AGt0 & AGt1 & p-value & NAGt0 & NAGt1 & p-value \\
\hline SGRQ total (IQR) & $35(18-55)$ & $29(15-47)$ & 0.022 & $34(25-59)$ & $38(18-54)$ & ns \\
\hline T0 SGRQ Symptoms score (IQR) & $30(19-54)$ & $18(13-34)$ & 0.0055 & $36(21-67)$ & $26(11-68)$ & ns \\
\hline T0 SGRQ Impact score (IQR) & $29(11-59)$ & $25(11-43)$ & 0.044 & $28(19-48)$ & $33(10-46)$ & ns \\
\hline T0 SGRQ Activity score (IQR) & $47(29-66)$ & $35(26-65)$ & ns & $49(30-73)$ & $44(31-65)$ & ns \\
\hline HAQ-MOD & $0.7(0.3-1.5)$ & $0.5(0.1-1.1)$ & ns & $1.1(0.3-2.5)$ & $1.1(0.4-2-0)$ & ns \\
\hline SF36 physical & $33(28-42)$ & $38(30-45)$ & ns & $25(21-38)$ & $30(21-42)$ & ns \\
\hline SF36 mental & $48(41-51)$ & $48(39-55)$ & ns & $30(22-39)$ & $31(23-41)$ & ns \\
\hline
\end{tabular}

SGRQ, Saint George's Respiratory Questionnaire; HAQ-MOD, modified Health Assessment Questionnaire for SSc; SF36, Short Form 36; AG, adherent group; NAG, non-adherent group; ns, not significant. 
trained with home-based PR, in order to strengthen the evidence up to include PR strategies in the SSc treatment guidelines.

\section{Key points}

- Dyspnea decreases SSc patients' quality of life (QoL).

- In SSc, Pulmonary Rehabilitation (PR) reduces breathlessness and disability, improving QoL.

- PR should be considered in the treatment strategy of SSc patients with dyspnea.

\section{References}

1. Solomon JJ, Olson AL, Fischer A, et al. Scleroderma lung disease. Eur Respir Rev 2013;22:6-19.

2. Spruit MA, Singh SJ, Garvey C, et al. An official American Thoracic Society/ European Respiratory Society statement: key concepts and advances in pulmonary rehabilitation. Am J Respir Crit Care Med 2013;188:e13-e64.

3. Raghu G, Collard HR, Egan JJ, et al. An official ATS/ERS/JRS/ALAT statement: idiopathic pulmonary fibrosis: evidence-based guidelines for diagnosis and management. Am J Respir Crit Care Med 2011;183:788-824.

4. Ferrer M, Villasante C, Alonso J, et al. Interpretation of quality of life scores from the St George's Respiratory Questionnaire. Eur Respir J 2002;19:405-13.

5. Jones PW. St. George's Respiratory Questionnaire: MCID. COPD 2005;2:75-9.

6. Pope J. Measures of systemic sclerosis (scleroderma): Health Assessment Questionnaire (HAQ) and Scleroderma HAQ (SHAQ), physician- and patient-rated global assessments, Symptom Burden Index (SBI), University of California, Los Angeles, Scleroderma Clinical Trials Consortium Gastrointestinal Scale (UCLA SCTC GIT) 2.0, Baseline Dyspnea Index (BDI) and Transition Dyspnea Index (TDI) (Mahler's Index), Cambridge Pulmonary Hypertension
Outcome Review (CAMPHOR), and Raynaud's Condition Score (RCS). Arthritis Care Res (Hoboken) 2011;63:S98-111.

7. Johnson SR, Hawker GA, Davis AM. The health assessment questionnaire disability index and scleroderma health assessment questionnaire in scleroderma trials: an evaluation of their measurement properties. Arthritis Rheum 2005;53:256-62.

8. McHorney CA, Ware JE, Lu JFR, Sherbourne CD. The MOS 36-Item Short-Form Health Survey (SF-36®): III. tests of data quality, scaling assumptions and reliability across diverse patient groups. Med Care 1994;32:40-66.

9. Holland AE. Exercise limitation in interstitial lung disease mechanisms, significance and therapeutic options. Chron Respir Dis 2010;7:101-11.

10. Rochester CL, Vogiatzis I, Holland AE, et al. An Official American Thoracic Society/European Respiratory Society Policy Statement: enhancing implementation, use, and delivery of pulmonary rehabilitation. Am J Respir Crit Care Med 2015;192:1373-86.

11. Spruit MA, Pitta F, Garvey C, et al. Differences in content and organisational aspects of pulmonary rehabilitation programmes. Eur Respir J 2014;43:1326-37.

12. Ryerson CJ, Berkeley J, Carrieri-Kohlman VL, et al. Depression and functional status are strongly associated with dyspnea in interstitial lung disease. Chest 2011;139:609-16.

13. Akhtar AA, Ali MA, Smith RP. Depression in patients with idiopathic pulmonary fibrosis. Chron Respir Dis 2013;10:127-33.

14. Ferrara G, Luppi F, Birring SS, et al. Best supportive care for idiopathic pulmonary fibrosis: current gaps and future directions. Eur Respir Rev 2018;27:170076.

15. McCarthy B, Casey D, Devane D, et al. Pulmonary rehabilitation for chronic obstructive pulmonary disease. Cochrane Database Syst Rev 2015;(2):CD003793.

16. Holland AE, Mahal A, Hill CJ, et al. Home-based rehabilitation for COPD using minimal resources: a randomised, controlled equivalence trial. Thorax 2017;72:57-65.

17. Lumetti F, Barone L, Alfieri C, et al. Quality of life and functional disability in patients with interstitial lung disease related to Systemic Sclerosis. Acta Biomed 2015;86:142-8. 